\title{
Ground-State Phase Diagram of Geometrically Frustrated Ising-Heisenberg Model on Doubly Decorated Planar Lattices
}

\author{
L. ČAnová, J. StrečKa, J. Dely And M. JAŠČUR
}

Department of Theoretical Physics and Astrophysics, Faculty of Science P.J. Šafárik University, Park Angelinum 9, 04001 Košice, Slovak Republic

Ground-state phase diagram of the mixed spin-1/2 and spin-1 IsingHeisenberg model on doubly decorated planar lattices is examined using the generalized decoration-iteration transformation. The main attention is devoted to the comparison of the ground-state properties of the quantum Ising-Heisenberg model and its semi-classical Ising analogue.

PACS numbers: 05.50.+q, 75.10.Hk, 75.10.Jm

\section{Introduction}

Frustrated quantum models have attracted a great research interest during the last three decades especially due to their extraordinary diverse ground-state behaviour, which often arises as a result of mutual interplay between the geometric frustration and quantum fluctuations [1]. Despite a considerable effort, the geometric frustration and its effect on magnetic properties of frustrated spin systems have not been fully elucidated, yet. Owing to this fact, exactly solvable geometrically frustrated models can serve as useful models for in-depth understanding of this phenomenon [2]. In this work, we shall provide the exact solution for the special class of geometrically frustrated Ising-Heisenberg planar models, which could be potentially helpful in the examination of the geometric frustration.

\section{Model system and its solution}

Let us consider the mixed spin-1/2 and spin-1 Ising-Heisenberg model on doubly decorated planar lattices as schematically depicted on the left-hand side of Fig. 1 for the case of doubly decorated square lattice. The total Hamiltonian of the model under investigation can be written in the form $\hat{\mathcal{H}}_{\mathrm{H}}=\sum_{k=1}^{N q / 2} \hat{\mathcal{H}}_{k}$, where the summation is carried out over all bonds of the original (undecorated) lattice 


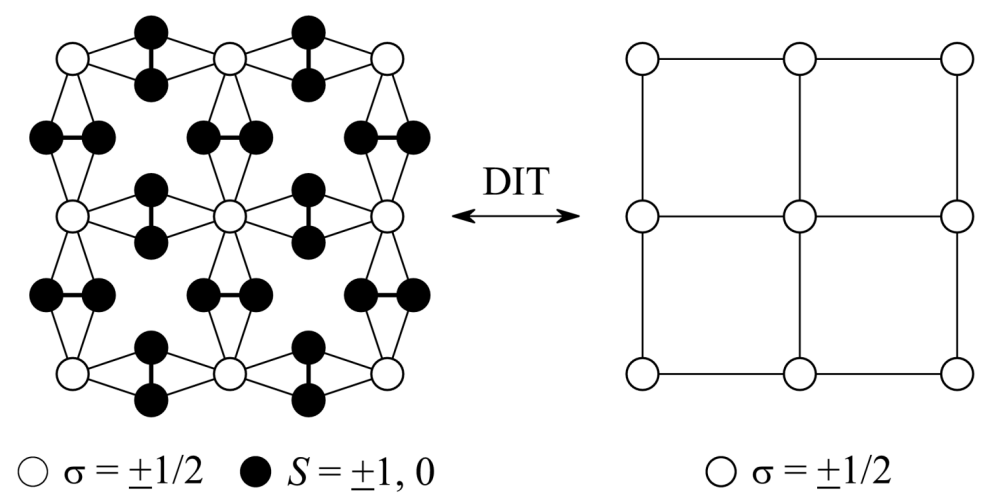

Fig. 1. Diagrammatic illustration of the used mapping transformation. The mixed spin-1/2 and spin-1 Ising-Heisenberg model on the doubly decorated square lattice is mapped on the simple spin-1/2 Ising model on the corresponding lattice. Empty (full) circles denote lattice positions of the Ising (Heisenberg) spins.

of $N$ atoms with the coordination number $q$. The bond Hamiltonian $\hat{\mathcal{H}}_{k}$ is given by the formula

$$
\begin{aligned}
\hat{\mathcal{H}}_{k}= & J_{\mathrm{H}}\left[\Delta\left(\hat{S}_{k 1}^{x} \hat{S}_{k 2}^{x}+\hat{S}_{k 1}^{y} \hat{S}_{k 2}^{y}\right)+\hat{S}_{k 1}^{z} \hat{S}_{k 2}^{z}\right]-D\left[\left(\hat{S}_{k 1}^{z}\right)^{2}+\left(\hat{S}_{k 2}^{z}\right)^{2}\right] \\
& +J_{\mathrm{I}}\left(\hat{S}_{k 1}^{z}+\hat{S}_{k 2}^{z}\right)\left(\hat{\sigma}_{k 1}^{z}+\hat{\sigma}_{k 2}^{z}\right),
\end{aligned}
$$

where $\hat{\sigma}_{k}^{z}$ and $\hat{S}_{k}^{\alpha}(\alpha=x, y, z)$ denote spatial components of the spin- $1 / 2$ and spin-1 operators, $J_{\mathrm{H}}, J_{\mathrm{I}}$ stand for the exchange interactions between the nearest-neighbour Heisenberg spins, the Heisenberg and Ising spins, respectively, $\Delta$ marks the $X X Z$ exchange anisotropy and $D$ denotes the single-ion anisotropy. Using the commutation rule between different bond Hamiltonians and applying the generalized decoration-iteration mapping transformation [3], one obtains the equality

$$
\mathcal{Z}_{\mathrm{IH}}\left(\beta, J_{\mathrm{H}}, J_{\mathrm{I}}, \Delta, D\right)=A^{N q / 2} \mathcal{Z}_{\mathrm{I}}(\beta, R)
$$

which establishes an exact mapping relationship between the partition function $\mathcal{Z}_{\mathrm{IH}}$ of the Ising-Heisenberg model and the partition function $\mathcal{Z}_{\mathrm{I}}$ of the spin-1/2 Ising model on the corresponding lattice (see Fig. 1). Let us notice that the mapping relation (2) is universal and valid regardless of the lattice topology or space dimensionality of the investigated system. Besides, it also allows direct calculation of all relevant physical quantities, which are useful for the understanding of the magnetic behaviour in the whole parameter space. In this regard, exact results for the Ising-Heisenberg model on several decorated planar lattices can be obtained, because of known exact solutions for partition functions of many spin-1/2 Ising planar lattices [3].

\section{Results and concluding remarks}

Now, let us take a closer look at the ground-state behaviour of the mixed-spin Ising-Heisenberg model with the antiferromagnetic (AF) exchange parame- 
ters $J_{\mathrm{I}}$ and $J_{\mathrm{H}}\left(J_{\mathrm{I}}>0, J_{\mathrm{H}}>0\right)$. Let us note that this sign choice closely relates to our endeavour to match the situation in the geometrically frustrated IsingHeisenberg planar models. Before discussing the results in detail, it is important to remark that the ground-state behaviour discussed below is rather general due to the universality of Eq. (2), since this mapping relation holds regardless of the lattice coordination number $q$.
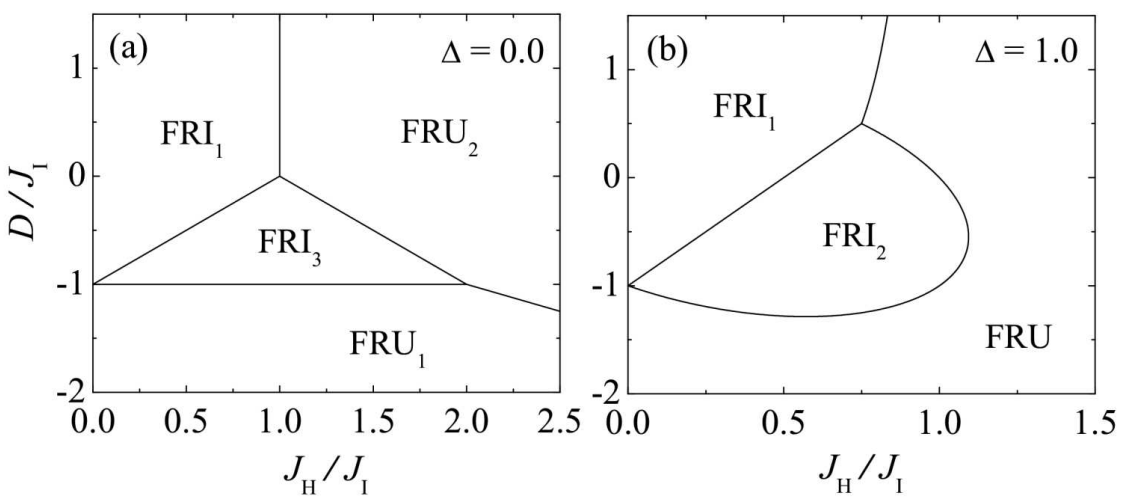

Fig. 2. Ground-state phase diagrams of the spin-1/2 and spin-1 Ising-Heisenberg planar model in the $J_{\mathrm{H}}-D$ space for $\Delta=0.0$ (a) and $\Delta=1.0$ (b).

For illustration, two ground-state phase diagrams are displayed in the $J_{\mathrm{H}}-D$ space in Fig. 2 for $\Delta=0.0$ and $\Delta=1.0$. As one can see, the mutual competition between the parameters $J_{\mathrm{H}}, J_{\mathrm{I}}, D$, and $\Delta$ gives rise to six possible ground states. Spin order to emerge within each sector of the phase diagrams can be unambiguously characterized by the wave functions

$$
\begin{aligned}
& \left|\mathrm{FRI}_{1}\right\rangle=\prod_{i=1}^{N}|-\rangle_{i} \prod_{k=1}^{N q / 2}|1,1\rangle_{k},\left|\mathrm{FRI}_{2}\right\rangle=\prod_{i=1}^{N}|-\rangle_{i} \prod_{k=1}^{N q / 2} \frac{1}{\sqrt{2}}\left(|1,0\rangle_{k}-|0,1\rangle_{k}\right) ; \\
& \left|\mathrm{FRI}_{3}\right\rangle=\prod_{i=1}^{N}|-\rangle_{i} \prod_{k=1}^{N q / 2}|1,0\rangle_{k} \text { or }\left|\mathrm{FRI}_{3}\right\rangle=\prod_{i=1}^{N}|-\rangle_{i} \prod_{k=1}^{N q / 2}|0,1\rangle_{k} ; \\
& |\mathrm{FRU}\rangle=\prod_{i=1}^{N}| \pm\rangle_{i} \prod_{k=1}^{N q / 2} \frac{1}{2}\left[a_{+}\left(|1,-1\rangle_{k}+|-1,1\rangle_{k}\right)-\sqrt{2} a_{-}|0,0\rangle_{k}\right],
\end{aligned}
$$

where $a_{ \pm}=\sqrt{1 \pm \delta^{-1}}, \delta^{2}=1+8\left(J_{\mathrm{H}} \Delta\right)^{2} /\left(J_{\mathrm{H}}+2 D\right)^{2}$. In above, the first product is taken over all Ising spins $\left(| \pm\rangle\right.$ stands for $\left.\sigma^{z}= \pm 1 / 2\right)$ and the second one runs over all Heisenberg spin pairs $\left(| \pm 1,0\rangle\right.$ is assigned to $\left.S^{z}= \pm 1,0\right)$. It is quite evident from the aforelisted eigenfunctions that $\mathrm{FRI}_{1}$ represents the semi-classically ordered ferrimagnetic phase with the antiparallel alignment between the nearestneighbour Ising and Heisenberg spins. Besides, another two ferrimagnetic phases $\mathrm{FRI}_{2}$ and $\mathrm{FRI}_{3}$ can be found in the quantum Ising-Heisenberg model and in its 
semi-classical Ising version, respectively. Both these phases appear as results of the competition between the AF exchange interaction $J_{\mathrm{H}}$ and the easy-plane singleion anisotropy $(D<0)$ and the only difference between them lies in the quantum entanglement that emerges just within $\mathrm{FRI}_{2}\left(\mathrm{FRI}_{3}\right.$ is classical ferrimagnetic phase without entanglement). Finally, several frustrated phases can be found in the ground state depending on whether the exchange anisotropy is zero or non-zero. If $\Delta \neq 0.0$, then the unique frustrated phase FRU can be detected as shown in Fig. $2 \mathrm{~b}$ for the particular case $\Delta=1.0$. In this phase, the Heisenberg spin pairs exhibit quantum entanglement of three spin states $|0,0\rangle$ and $| \pm 1, \mp 1\rangle$, whereas their probability amplitudes depend on a mutual ratio between $J_{\mathrm{H}}, \Delta$, and $D$ according to Eq. (5). If $\Delta=0.0$, the ground-state phase diagram consists of two different frustrated phases FRU $\mathrm{F}_{1}$ and $F R U_{2}$ instead of unique FRU one. In the former frustrated phase $\mathrm{FRU}_{1}$, all Heisenberg spin pairs reside in the "non-magnetic" $|0,0\rangle$ spin state, while in the latter phase $\mathrm{FRU}_{2}$, they reside in one of two intrinsic $\mathrm{AF}$ spin states. Let us note that both the frustrated phases $\mathrm{FRU}_{1}$ and $\mathrm{FRU}_{2}$ can be regarded as limiting cases of the frustrated phase FRU.

In conclusion, the investigated Ising-Heisenberg model exhibits an interesting ground-state behaviour including the peculiar geometric spin frustration. The more detailed examination concerned with the finite-temperature phase diagram of this spin system will be presented in the near future.

\section{Acknowledgments}

This work was supported under the scientific grants VEGA 1/2009/05, LPP0107-06, and VVGS PF 02/2007/F.

\section{References}

[1] H.T. Diep, Magnetic Systems with Competing Interactions, World Sci., Singapore 1994.

[2] J. Strečka, M. Jaščur, Phys. Status Solidi B 233, R12 (2004).

[3] I. Syozi, in: Phase Transitions and Critical Phenomena, Vol. 1, Eds. C. Domb, M.S. Green, Academic Press, New York 1972, p. 322. 